\title{
The Church-Rosser Languages Are the Deterministic Variants of the Growing Context-Sensitive Languages
}

\author{
Gundula Niemann and Friedrich Otto \\ Fachbereich Mathematik/Informatik, Universität Kassel, D-34109 Kassel \\ e-mail: <niemann, otto>otheory .informatik, uni-kassel.de
}

\begin{abstract}
The growing context-sensitive languages have been classified through the shrinking two-pushdown automaton, the deterministic version of which characterizes the class of generalized Church-Rosser languages (Buntrock and Otto 1995). Exploiting this characterization we prove that this latter class coincides with the class of Church-Rosser languages that was introduced by McNaughton, Narendran, and Otto (1988). Based on this result several open problems of McNaughton et al can be answered.
\end{abstract}

\section{Introduction}

If $R$ is a finite and length-reducing string-rewriting system on some finite alphabet $\Sigma$, then there exists a linear-time algorithm that, given a string $w \in \Sigma^{*}$ as input, computes an irreducible descendant $w_{0}$ of $w$ with respect to the reduction relation $\rightarrow_{R}^{*}$ that is induced by $R[2,3]$. If, in addition, the system $R$ is confluent, then the irreducible descendant $w_{0}$ is uniquely determined by $w$. Hence, in this situation two strings $u$ and $v$ are congruent modulo the Thue congruence $\leftrightarrow_{R}^{*}$ induced by $R$ if and only if their respective irreducible descendants $u_{0}$ and $v_{0}$ coincide. Thus, the word problem for a finite, length-reducing, and confluent string-rewriting system is decidable in linear time.

Motivated by this result McNaughton, Narendran, and Otto [11] introduced the notion of a Church-Rosser language. A Church-Rosser language $L \subseteq \Sigma^{*}$ is given through a finite, length-reducing, and confluent string-rewriting system $R$ on some alphabet $\Gamma$ properly containing $\Sigma$, two irreducible strings $t_{1}, t_{2} \in$ $(\Gamma \backslash \Sigma)^{*}$, and an irreducible letter $Y \in \Gamma \backslash \Sigma$ satisfying the following condition for all strings $w \in \Sigma^{*}: w \in L$ if and only if $t_{1} w t_{2} \rightarrow_{R}^{*} Y$. Hence, the membership problem for a Church-Rosser language is decidable in linear time, and so the class CRL of Church-Rosser languages is contained in the class CSL of contextsensitive languages.

On the other hand, the class CRL contains the class DCFL of deterministic context-free languages, and it contains some languages that are not even contextfree [11]. Hence, the class CRL can be seen as an extension of the class DCFL that preserves the linear-time decidability of the membership problem. As such it is certainly an interesting language class. 
Accordingly, McNaughton et al established some closure properties for the class CRL, but it remained open whether the class CRL is closed under the operation of complementation. Accordingly, they introduced the class of ChurchRosser decidable languages CRDL, which still contains the class DCFL and which is closed under complementation. Also it remained open at the time whether or not every context-free language is a Church-Rosser language, although it was conjectured that the linear language $L_{0}:=\left\{w w^{\sim} \mid w \in\{a, b\}^{*}\right\}$ is not a ChurchRosser language. Here $w^{\sim}$ denotes the reversal of the string $w$.

After their introduction the Church-Rosser languages did not receive much attention until another, seemingly unrelated development had taken place. Dahlhaus and Warmuth [8] considered the class GCSL of growing context-sensitive languages. These languages are generated by context-sensitive grammars each production rule of which is strictly length-increasing. They proved that these languages have membership problems that are decidable in polynomial time. Although it might appear from the definition that GCSL is not an interesting class of languages, Buntrock and Loryś showed that GCSL is an abstract family of languages [5], that is, this class of languages is closed under union, concatenation, iteration, intersection with regular languages, $\varepsilon$-free homomorphisms, and inverse homomorphisms. Exploiting these closure properties Buntrock and Loryś characterized the class GCSL through various other classes of grammars that are less restricted $[5,6]$.

Using these grammars Buntrock and Otto [7] obtained a characterization of the class GCSL by a nondeterministic machine model, the so-called shrinking pushdown automaton with two pushdown stores (sTPDA). The input for such a machine is provided as the initial contents of one of the pushdown stores, and it accepts either by final state or (equivalently) by empty pushdown stores. A positive weight is assigned to each tape symbol and each internal state symbol of the machine. By adding up the weights this gives a weight for each configuration. Now it is required that the weight of the actual configuration decreases with each step of the machine. It is with respect to these weights that the two-pushdown automaton is called shrinking.

Since the sTPDA is a nondeterministic device, it was only natural to consider the class of languages that are accepted by the deterministic variant of it. As it turned out the deterministic sTPDA accept exactly the so-called generalized Church-Rosser languages, which are obtained from the Church-Rosser languages by admitting finite, weight-reducing, and confluent string-rewriting systems in the definition [7]. Thus, the class GCRL of generalized Church-Rosser languages coincides with the class of 'deterministic growing context-sensitive languages.' In particular, it follows that this class is closed under complementation. Further, Buntrock and Otto concluded from this result that the language classes CFL and GCRL, and therewith the classes CFL and CRL, are indeed incomparable under set inclusion. Since CFL is contained in GCSL, it follows that GCRL is properly contained in the class GCSL, that is, we obtain the following chain of (proper) inclusions:

$\mathrm{DCFL} \subset \mathrm{CRDL} \subseteq \mathrm{CRL} \subseteq \mathrm{GCRL} \subset \mathrm{GCSL} \subset \mathrm{CSL}$ 
where it was left open whether or not the two inclusions CRDL $\subseteq$ CRL $\subseteq$ GCRL are proper.

Here we show that the three language classes CRDL, CRL, and GCRL coincide. Our proof makes use of the above-mentioned characterization of the generalized Church-Rosser languages through the deterministic sTPDA. We will prove that each language that is accepted by some deterministic sTPDA is actually a Church-Rosser decidable language. Hence, GCRL $\subseteq$ CRDL implying that the three classes above actually coincide. Hence, the class of Church-Rosser languages can be characterized as the class of deterministic growing contextsensitive languages.

It remains to determine the closure properties of this class of languages. The closure under the operation of taking the complement follows from the above characterization. Recently, Otto, Katsura, and Kobayashi [12] proved that the class of Church-Rosser languages is a basis for the recursively enumerable (r.e.) languages. Here, a class of languages $\mathbb{C}$ is called a basis for the r.e. languages, if, for each r.e. language $L \subseteq \Sigma^{*}$, there exists a language $C \in \mathbb{C}$ on some alphabet $\Gamma$ strictly containing $\Sigma$ such that $L=\pi_{\Sigma}(C)$, where $\pi_{\Sigma}$ denotes the canonical projection from $\Gamma^{*}$ onto $\Sigma^{*}$. It follows that the class CRL is not closed under morphisms.

This paper is organized as follows. In Section 2 we introduce the necessary notation regarding string-rewriting systems and restate the definitions of the various classes of Church-Rosser languages. In the next section we introduce the shrinking two-pushdown automaton and restate some results from Buntrock and Otto [7]. In addition we prove a technical result for this type of automaton. Then in Section 4 we prove the announced main result, and in the next section we summarize the known closure and non-closure properties of the class CRL. In the final section we review our results and draw some easy consequences.

\section{The Church-Rosser Languages}

Here we restate the main definitions and establish notation regarding the various classes of Church-Rosser languages. For additional information concerning the notions introduced the reader is asked to consult the literature, where [3] serves as our main reference concerning the theory of string-rewriting systems, and [10] is our main reference for formal language and automata theory.

Let $\Sigma$ be a finite alphabet. Then $\Sigma^{*}$ denotes the set of strings over $\Sigma$ including the empty string $\varepsilon$, and $\Sigma^{+}:=\Sigma^{*} \backslash\{\varepsilon\}$. A function $\varphi: \Sigma \rightarrow \mathbb{N}_{+}$is called a weight-function. Its extension to $\Sigma^{*}$, which we will also denote by $\varphi$, is defined inductively through $\varphi(\varepsilon):=0$ and $\varphi(w a):=\varphi(w)+\varphi(a)$ for all $w \in \Sigma^{*}$ and $a \in \Sigma$. A particular weight-function is the length-function $||:. \Sigma \rightarrow \mathbb{N}_{+}$, which assigns each letter the weight (length) 1.

A string-rewriting system $R$ on $\Sigma$ is a subset of $\Sigma^{*} \times \Sigma^{*}$. An element $(\ell, r) \in R$ is called a rewrite rule or simply a rule, and it will usually be written as $(\ell \rightarrow r)$. A string-rewriting system $R$ induces several binary relations on $\Sigma^{*}$, the simplest 
of which is the single-step reduction relation

$$
\rightarrow_{R}:=\left\{(u \ell v, u r v) \mid u, v \in \Sigma^{*},(\ell \rightarrow r) \in R\right\}
$$

Its reflexive and transitive closure is the reduction relation $\rightarrow_{R}^{*}$ induced by $R$, and its reflexive, symmetric, and transitive closure $\leftrightarrow_{R}^{*}$ is the Thue congruence generated by $R$.

If $u \rightarrow_{R}^{*} v$, then $u$ is an ancestor of $v$, and $v$ is a descendant of $u$. If there is no $v \in \Sigma^{*}$ such that $u \rightarrow_{R} v$ holds, then the string $u$ is called irreducible $(\bmod R)$. By $\operatorname{IRR}(R)$ we denote the set of all irreducible strings. If $R$ is finite, then $\operatorname{IRR}(R)$ is obviously a regular language.

The string-rewriting system $R$ is called

- length-reducing if $|\ell|>|r|$ holds for each rule $(\ell \rightarrow r) \in R$,

- weight-reducing if there exists a weight-function $\varphi$ such that $\varphi(\ell)>\varphi(r)$ holds for each rule $(\ell \rightarrow r) \in R$,

- confluent if, for all $u, v, w \in \Sigma^{*}, u \rightarrow_{R}^{*} v$ and $u \rightarrow_{R}^{*} w$ imply that $v$ and $w$ have a common descendant.

If a string-rewriting system $R$ is weight-reducing, then it allows no infinite reduction sequence of the form $w_{0} \rightarrow_{R} w_{1} \rightarrow_{R} \ldots$; indeed, if $w_{0} \rightarrow_{R} w_{1} \rightarrow_{R}$ $\ldots \rightarrow_{R} w_{m}$, then $m \leq \varphi\left(w_{0}\right)$. If, in addition, $R$ is confluent, then each string $w \in \Sigma^{*}$ has a unique irreducible descendant $w_{0} \in \operatorname{IRR}(R)$. Actually, in this situation $u \leftrightarrow_{R}^{*} v$ if and only if $u_{0}=v_{0}$. Since $u_{0}$ can be determined from $u$ in linear time, this shows that the Thue congruence $\leftrightarrow_{R}^{*}$ is decidable in linear time for each finite, weight-reducing, and confluent string-rewriting system.

\section{Definition 1.}

(a) A language $L \subseteq \Sigma^{*}$ is a Church-Rosser language (CRL) if there exist an alphabet $\Gamma \supsetneq \Sigma$, a finite, length-reducing, confluent string-rewriting system $R$ on $\Gamma$, two strings $t_{1}, t_{2} \in(\Gamma \backslash \Sigma)^{*} \cap \operatorname{IRR}(R)$, and a letter $Y \in(\Gamma \backslash \Sigma) \cap$ $\operatorname{IRR}(R)$ such that, for all $w \in \Sigma^{*}, t_{1} w t_{2} \rightarrow_{R}^{*} Y$ if and only if $w \in L$.

(b) A language $L \subseteq \Sigma^{*}$ is a Church-Rosser decidable language (CRDL) if it is a Church-Rosser language, and there exists a letter $N \in(\Gamma \backslash \Sigma) \cap \operatorname{IRR}(R)$ such that, for all $w \in \Sigma^{*}, t_{1} w t_{2} \rightarrow_{R}^{*} N$ if and only if $w \notin L$.

(c) A language $L \subseteq \Sigma^{*}$ is a generalized Church-Rosser language (GCRL) if there exist an alphabet $\Gamma \supsetneq \Sigma$, a finite, weight-reducing, confluent stringrewriting system $R$ on $\Gamma$, two strings $t_{1}, t_{2} \in(\Gamma \backslash \Sigma)^{*} \cap \operatorname{IRR}(R)$ and a letter $Y \in(\Gamma \backslash \Sigma) \cap \operatorname{IRR}(R)$ such that, for all $w \in \Sigma^{*}, t_{1} w t_{2} \rightarrow_{R}^{*} Y$ if and only if $w \in L$.

Analogously to (b) the class of generalized Church-Rosser decidable languages could be defined, but the results of Buntrock and Otto [7] imply that this class coincides with the class GCRL of generalized Church-Rosser languages. 


\section{Shrinking Two-Pushdown Automata}

In [7] Buntrock and Otto introduce the following type of automaton in order to characterize the class GCSL of growing context-sensitive languages.

\section{Definition 2.}

(a) A two-pushdown automaton (TPDA) is a nondeterministic automaton with two pushdown stores. It is defined as a 7 -tuple $M=\left(Q, \Sigma, \Gamma, \delta, q_{0}, \perp, F\right)$, where

- $Q$ is the finite set of states,

$-\Sigma$ is the finite input alphabet,

$-\Gamma$ is the finite tape alphabet with $\Gamma \supsetneq \Sigma$ and $\Gamma \cap Q=\emptyset$,

$-q_{0} \in Q$ is the initial state,

- $\perp \in \Gamma \backslash \Sigma$ is the bottom marker of pushdown stores,

$-F \subseteq Q$ is the set of final (or accepting) states, and

$-\delta: Q \times \Gamma \times \Gamma \rightarrow 2^{Q \times \Gamma^{*} \times \Gamma^{*}}$ is the transition relation, where $\delta(q, a, b)$ is a finite set for each triple $(q, a, b) \in Q \times \Gamma \times \Gamma$.

$M$ is a deterministic two-pushdown automaton (DTPDA), if $\delta$ is a (partial) function from $Q \times \Gamma \times \Gamma$ into $Q \times \Gamma^{*} \times \Gamma^{*}$.

(b) A configuration of a (D)TPDA $M$ is described as uqv with $q \in Q$ and $u, v \in$ $\Gamma^{*}$, where $u$ is the contents of the first pushdown store with the first letter of $u$ at the bottom and the last letter of $u$ at the top, $q$ is the current state, and $v$ is the contents of the second pushdown store with the last letter of $v$ at the bottom and the first letter of $v$ at the top. $M$ induces a computation relation $\vdash_{M}^{*}$ on the set of configurations, which is the reflexive, transitive closure of the single-step computation relation $\vdash_{M}$ (see,e.g., [10]). For an input string $w \in \Sigma^{*}$, the corresponding initial configuration is $\perp q_{0} w \perp . M$ accepts by empty pushdown stores:

$$
N(M):=\left\{w \in \Sigma^{*} \mid \exists q \in Q: \perp q_{0} w \perp \vdash_{M}^{*} q\right\} .
$$

(c) $A$ (D)TPDA $M$ is called shrinking if there exists a weight function $\varphi: Q \cup$ $\Gamma \rightarrow \mathbb{N}_{+}$such that, for all $q \in Q$ and $a, b \in \Gamma$, if $(p, u, v) \in \delta(q, a, b)$, then $\varphi(u p v)<\varphi(a q b)$. By sTPDA and sDTPDA we denote the corresponding classes of shrinking automata.

Thus, if $M$ is a shrinking TPDA with weight-function $\varphi$, then $\varphi\left(u_{1} q_{1} v_{1}\right)>$ $\varphi\left(u_{2} q_{2} v_{2}\right)$ holds for all configurations $u_{1} q_{1} v_{1}$ and $u_{2} q_{2} v_{2}$ of $M$ that satisfiy $u_{1} q_{1} v_{1} \vdash_{M} u_{2} q_{2} v_{2}$. Observe that the input is provided to a TPDA as the initial contents of its second pushdown store, and that in order to accept a TPDA is required to empty its pushdown stores. Thus, it is forced to consume the input completely. Using standard techniques from automata theory it can be shown that, for a (shrinking) (D)TPDA $M=\left(Q, \Sigma, \Gamma, \delta, q_{0}, \perp, F\right)$, we may require that the special symbol $\perp$ can only occur at the bottom of a pushdown store, and that no other symbol can occur at that place. 
From the definition of the transition relation $\delta$ we see that $M$ halts immediately whenever one of its pushdown stores is emptied. Because of the above property this happens if and only if a transition of the form $(q, a, \perp) \mapsto\left(q^{\prime}, \alpha, \varepsilon\right)$ or $(q, \perp, b) \mapsto\left(q^{\prime}, \varepsilon, \beta\right)$ is performed. Thus, we can assume without loss of generality that, if $M$ does accept on input $w \in \Sigma^{*}$, then $\perp q_{0} w \perp \vdash_{M}^{*} q$ for some $q \in F$, and if $M$ does not accept on input $w \in \Sigma^{*}$, then $\perp q_{0} w \perp \vdash_{M}^{*} \perp q$ for some $q \in F$, that is, even in this situation $M$ empties its second pushdown store completely and only leaves the bottom marker on its first pushdown store before it halts. Hence, all the halting and accepting configurations of $M$ are of the form $q$, where $q \in F$, and all the halting and rejecting configurations of $M$ are of the form $\perp q$, where $q \in F$. In addition, we can assume that $M$ only has a single halting state.

Buntrock and Otto established the following characterization for the classes of languages that are accepted by nondeterministic or deterministic shrinking TPDAs, respectively.

\section{Proposition 3. [7]}

(a) A language is accepted by some shrinking TPDA if and only if it is growing context-sensitive.

(b) A language is accepted by some shrinking DTPDA if and only if it is a generalized Church-Rosser language.

A detailed presentation of the class GCSL of growing context-sensitive languages can be found in Buntrock's Habilitationsschrift [4]. The above proposition shows that the generalized Church-Rosser languages can be interpreted as the deterministic variants of the growing context-sensitive languages.

We close this section with a technical lemma on shrinking TPDA that we will need in the next section to prove our main result.

Lemma 4. Let $M$ be a TPDA that is shrinking with respect to the weightfunction $\varphi$. Then there exists a TPDA $M^{\prime}$ accepting the same language as $M$ such that $M^{\prime}$ is deterministic, if $M$ is, and $M^{\prime}$ is shrinking with respect to a weight-function $\psi$ that satisfies the following condition:

(*) Whenever $u_{1} q_{1} v_{1}$ and $u_{2} q_{2} v_{2}$ are configurations of $M^{\prime}$ such that $u_{1} q_{1} v_{1} \vdash_{M^{\prime}}$ $u_{2} q_{2} v_{2}$, then $\psi\left(u_{1} q_{1} v_{1}\right)-\psi\left(u_{2} q_{2} v_{2}\right)=1$.

Proof. Let $M=\left(Q, \Sigma, \Gamma, \delta, q_{0}, \perp, F\right)$ be a TPDA that is shrinking with respect to the weight-function $\varphi: Q \cup \Gamma \rightarrow \mathbb{N}_{+}$, that is, $\varphi(a q b)-\varphi(u p v)>0$ for all $q \in Q, a, b \in \Gamma$, and $(p, u, v) \in \delta(q, a, b)$. We construct a TPDA $M^{\prime}:=$ $\left(Q^{\prime}, \Sigma, \Gamma, \delta^{\prime}, q_{0}, \perp, F\right)$ and a weight-function $\psi: Q^{\prime} \cup \Gamma \rightarrow \mathbb{N}_{+}$as follows.

First we number the instructions of $M$, that is, the lines in the table describing the transition relation $\delta$, from 1 to $m$. For each $i \in\{1, \ldots, m\}$, let the $i$-th instruction of $M$ be denoted as $\left(p_{i}, u_{i}, v_{i}\right) \in \delta\left(q_{i}, a_{i}, b_{i}\right)$, and let $\gamma_{i}:=$ $\varphi\left(a_{i} q_{i} b_{i}\right)-\varphi\left(u_{i} p_{i} v_{i}\right)$. 
If $\gamma_{i}=1$, then take $Q_{i}^{\prime}:=\emptyset$ and add the transition $\left(q_{i}, a_{i}, b_{i}\right) \rightarrow\left(p_{i}, u_{i}, v_{i}\right)$ to $\delta^{\prime}$. If $\gamma_{i}>1$, then take $Q_{i}^{\prime}:=\left\{q_{i, 1}, \ldots, q_{i, \gamma_{i}-1}\right\}$, where $q_{i, 1}, \ldots, q_{i, \gamma_{i}-1}$ are $\gamma_{i}-1$ new states, and add the following transitions to $\delta^{\prime}$ :

$$
\begin{array}{ll}
\left(q_{i}, a_{i}, b_{i}\right) & \rightarrow\left(q_{i, 1}, a_{i}, b_{i}\right) \\
\left(q_{i, j}, a_{i}, b_{i}\right) & \rightarrow\left(q_{i, j+1}, a_{i}, b_{i}\right), j=1, \ldots, \gamma_{i}-2, \\
\left(q_{i, \gamma_{i}-1}, a_{i}, b_{i}\right) & \rightarrow\left(p_{i}, u_{i}, v_{i}\right) .
\end{array}
$$

Finally, let $Q^{\prime}:=Q \cup \bigcup_{i=1}^{m} Q_{i}^{\prime}$, let $\delta^{\prime}$ consist of all the transitions introduced so far, and define a preliminary weight-function $\psi^{\prime}: Q^{\prime} \cup \Gamma \rightarrow \mathbb{Z}$ as follows:

$$
\begin{aligned}
& \psi^{\prime}(a):=\varphi(a) \quad \text { for all } a \in \Gamma, \\
& \psi^{\prime}\left(q_{i}\right):=\varphi\left(q_{i}\right) \quad \text { for all } q_{i} \in Q, \\
& \psi^{\prime}\left(q_{i, j}\right):=\varphi\left(q_{i}\right)-j \text { for all } i \in\{1, \ldots, m\} \text { and } j \in\left\{1, \ldots, \gamma_{i}-1\right\} .
\end{aligned}
$$

It is easily verified that $\psi^{\prime}\left(u_{1} q_{1} v_{1}\right)-\psi^{\prime}\left(u_{2} q_{2} v_{2}\right)=1$ holds for all configurations $u_{1} q_{1} v_{1}$ and $u_{2} q_{2} v_{2}$ of $M^{\prime}$ that satisfy $u_{1} q_{1} v_{1} \vdash_{M^{\prime}} u_{2} q_{2} v_{2}$. Unfortunately, $\psi^{\prime}$ may not be an acceptable weight-function, since $\psi^{\prime}\left(q_{i, j}\right)$ could be a negative number for some choices of $i$ and $j$.

To correct this problem let $\mu:=\min \left\{\psi^{\prime}\left(p^{\prime}\right) \mid p^{\prime} \in Q^{\prime}\right\}$. If $\mu<0$, then choose $\psi\left(q^{\prime}\right):=\psi^{\prime}\left(q^{\prime}\right)+|\mu|+1$ for all $q^{\prime} \in Q^{\prime}$, otherwise, let $\psi\left(q^{\prime}\right):=\psi^{\prime}\left(q^{\prime}\right)$ for all $q^{\prime} \in Q^{\prime}$. Also choose $\psi(a):=\psi^{\prime}(a)$ for all $a \in \Gamma$. Then $\psi: Q^{\prime} \cup \Gamma \rightarrow \mathbb{N}_{+}$is a weight-function such that $\psi\left(u_{1} q_{1} v_{1}\right)-\psi\left(u_{2} q_{2} v_{2}\right)=1$ holds for all configurations $u_{1} q_{1} v_{1}$ and $u_{2} q_{2} v_{2}$ of $M^{\prime}$ that satisfy $u_{1} q_{1} v_{1} \vdash_{M^{\prime}} u_{2} q_{2} v_{2}$.

It is easily seen that $N\left(M^{\prime}\right)=N(M)$ and that $M^{\prime}$ is deterministic, if $M$ is deterministic.

Thus, in the following we can always assume that in each step of a sTPDA the weight of the actual configuration decreases by 1 . Hence, if $u_{1} q_{1} v_{1}$ and $u_{2} q_{2} v_{2}$ are configurations of an STPDA $M$ with weight-function $\varphi$ such that $u_{1} q_{1} v_{1} \vdash_{M}^{k}$ $u_{2} q_{2} v_{2}$ for some $k \in \mathbb{N}$, then $\varphi\left(u_{1} q_{1} v_{1}\right)-\varphi\left(u_{2} q_{2} v_{2}\right)=k$.

\section{The Main Result}

From the definitions we know that CRDL $\subseteq$ CRL $\subseteq$ GCRL holds. Here we prove that also GCRL $\subseteq$ CRDL holds, thus showing that the three classes actually coincide.

Theorem 5. GCRL $\subseteq$ CRDL.

Proof. Let $L \subseteq \Sigma^{*}$ be a generalized Church-Rosser language. By Proposition 3(b) there exist a DTPDA $M=\left(Q, \Sigma, \Gamma, \delta, q_{0}, \perp, F\right)$ and a weight-function $\varphi$ such that $N(M)=L$, where $M$ is shrinking with respect to $\varphi$. As observed in the previous section we can assume the following: 
(i) Each non-halting configuration of $M$ is of the form $\perp u q v \perp$ for some $u, v \in$ $(\Gamma \backslash\{\perp\})^{*}$ and $q \in(Q \backslash F)$.

(ii) $F=\left\{q_{f}\right\}$, that is, $M$ has a single halting state only.

(iii) The only accepting and halting configuration of $M$ that is reachable from an initial configuration is the configuration $q_{f}$.

(iv) The only non-accepting and halting configuration of $M$ that is reachable from an initial configuration is the configuration $\perp q_{f}$.

(v) If $u_{1} q_{1} v_{1} \vdash_{M} u_{2} q_{2} v_{2}$, then $\varphi\left(u_{1} q_{1} v_{1}\right)-\varphi\left(u_{2} q_{2} v_{2}\right)=1$ (Lemma 4).

Let \# be a new symbol. We define a morphism $h:(\Gamma \cup Q)^{*} \rightarrow(\Gamma \cup Q \cup\{\#\})^{*}$ by taking $h(a):=a \#^{\varphi(a)-1}$ for all $a \in \Gamma \cup Q$. Then $|h(w)|=\varphi(w)$ for all $w \in(\Gamma \cup Q)^{*}$, and $h(\Gamma \cup Q) \subseteq(\Gamma \cup Q \cup\{\#\})^{+}$is a prefix code. Thus, the morphism $h:(\Gamma \cup Q)^{*} \rightarrow(\Gamma \cup Q \cup\{\#\})^{*}$ is an injective mapping. Further, let $\mu:=\max \{\varphi(a) \mid a \in \Gamma \cup Q\}$ denote the maximal weight of any letter from $\Gamma \cup Q$.

In order to show that the language $L$ is actually Church-Rosser decidable, we now construct a finite, length-reducing, and confluent string-rewriting system $R$ on some finite alphabet $\Delta \supsetneq \Sigma$ that will witness this fact. Essentially $R$ will simulate the computations of the SDTPDA $M$. However, this cannot be a straightforward simulation, since $R$ is length-reducing, while $M$ is shrinking only with respect to the weight-function $\varphi$. Therefore we would like to replace a configuration $\perp u q v \perp$ of $M$ by the string $h(\perp u q v \perp)$. Since this replacement increases the length of the string considered, we need to compress the resulting string by combining several letters into a single new letter. This, however, creates another problem. If $\perp u_{1} q_{1} v_{1} \perp \vdash_{M} \perp u_{2} q_{2} v_{2} \perp$, then by (v) $\left|h\left(\perp u_{1} q_{1} v_{1} \perp\right)\right|-1=\left|h\left(\perp u_{2} q_{2} v_{2}\right)\right|$, but for the compressed forms of the strings $h\left(\perp u_{1} q_{1} v_{1} \perp\right)$ and $h\left(\perp u_{2} q_{2} v_{2} \perp\right)$ the length might be the same. To overcome this problem we choose the fixed rate of compression $2 \mu$, and simulate $2 \mu$ steps of $M$ through a single application of a rule of $R$. If $\perp u_{1} q_{1} v_{1} \perp \vdash_{M}^{2 \mu} \perp u_{2} q_{2} v_{2} \perp$, then $\left|h\left(\perp u_{1} q_{1} v_{1} \perp\right)\right|-2 \mu=\left|h\left(\perp u_{2} q_{2} v_{2} \perp\right)\right|$, and hence, if $\gamma_{1}$ and $\gamma_{2}$ are the compressed forms of $h\left(\perp u_{1} q_{1} u_{1} \perp\right)$ and $h\left(\perp u_{2} q_{2} v_{2} \perp\right)$, respectively, then $\left|\gamma_{1}\right|-1=\frac{\left|\boldsymbol{h}\left(\perp u_{1} q_{1} v_{1} \perp\right)\right|-2 \mu}{2 \mu}=\frac{\left|\boldsymbol{h}\left(\perp \boldsymbol{u}_{2} q_{2} v_{2} \perp\right)\right|}{2 \mu}=\left|\gamma_{2}\right|$.

To perform this construction we first determine the alphabet $\Delta$. Let $\bar{\Gamma} \cup\{\overline{\#}\}$ be a new alphabet that is in 1-to-1 correspondence to $\Gamma \cup\{\#\}$, and let $-: \Gamma \cup$ $\{\#\} \rightarrow \bar{\Gamma} \cup\{\overline{\#}\}$ denote this correspondence. Further, define four new alphabets as follows:

$$
\begin{aligned}
& A_{\leq}:=\left\{a_{w} \mid w \in(\Gamma \cup\{\#\})^{*} \text { and } 1 \leq|w| \leq \mu\right\}, \\
& A \quad:=\left\{a_{w} \mid w \in(\Gamma \cup\{\#\})^{*} \text { and }|w|=2 \mu\right\}, \\
& \bar{A}:=\left\{a \bar{w} \mid \bar{w} \in(\bar{\Gamma} \cup\{\overline{\#}\})^{*} \text { and }|\bar{w}|=2 \mu\right\}, \text { and } \\
& A_{Q}:=\left\{a_{\bar{u} q v} \mid \bar{u} \in(\bar{\Gamma} \cup\{\overline{\#}\})^{*}, q \in Q, v \in(\Gamma \cup\{\#\})^{*} \text { and }|\bar{u} q v|=2 \mu\right\} .
\end{aligned}
$$

Thus, each letter $a_{w} \in A_{\leq} \cup A \cup \bar{A} \cup A_{Q}$ represents a string $w$ of length at most $2 \mu$. Finally, we take $\Delta:=\Sigma \cup\left\{q_{0}, \bar{I}, \perp, Y, N\right\} \cup A_{\leq} \cup A \cup \bar{A} \cup A_{Q}$, where we assume that all the subalphabets displayed are pairwise disjoint.

To simplify the following considerations we define a morphism

$$
\pi:\left(A_{\leq} \cup A \cup \bar{A} \cup A_{Q}\right)^{*} \rightarrow(\Gamma \cup Q \cup\{\#\})^{*}
$$


through the following mapping:

$$
a \mapsto\left\{\begin{array}{l}
w, \quad \text { if } a=a_{w} \in A_{\leq} \cup A, \\
w, \quad \text { if } a=a_{\bar{w}} \in \bar{A}, \\
u q v, \text { if } a=a_{\bar{u} q v} \in A_{Q} .
\end{array}\right.
$$

Thus, $\pi$ replaces each letter $a \in A_{\leq} \cup A \cup \bar{A} \cup A_{Q}$ by the string it represents, where in addition each factor $\bar{u} \in(\bar{T} \cup\{\overline{\#}\})^{+}$is replaced by the corresponding string $u \in(\Gamma \cup\{\#\})^{+}$.

The string-rewriting system $R$ will consist of four subsystems $R_{0}, R_{1}, R_{2}$, and $R_{3}$.

(0) The subsystem $R_{0}$ is used to take care of those inputs $w \in \Sigma^{*}$ for the sDTPDA $M$ that are short:

$$
\begin{aligned}
R_{0} & :=\left\{I q_{0} w \perp \rightarrow Y \mid w \in \Sigma^{*}, \varphi(w \perp) \leq 4 \mu, \text { and } w \in L\right\} \\
& \cup\left\{I q_{0} w \perp \rightarrow N \mid w \in \Sigma^{*}, \varphi(w \perp) \leq 4 \mu, \text { and } w \notin L\right\} .
\end{aligned}
$$

Obviously, $R_{0}$ is a finite system containing only length-reducing rules, and there are no non-trivial overlaps between the left-hand sides of the rules of $\boldsymbol{R}_{\mathbf{0}}$.

(1) The subsystem $R_{1}$ transforms the description $I_{q_{0}} w \perp$ of an initial configuration $\perp q_{0} w \perp$ of $M$ into a compressed form $c \in \bar{A}^{*} \cdot A_{Q} \cdot A^{*}$, if $w$ is sufficiently long. It consists of three parts.

(1.1) $R_{1,1}:=\left\{w \perp \rightarrow \alpha^{\prime} \alpha_{1} \alpha_{2} \mid w=a v \in \Sigma^{*}\right.$ for some $a \in \Sigma$ such that

$$
\begin{aligned}
& \varphi(v \perp) \leq 4 \mu<\varphi(w \perp) \leq 5 \mu, \alpha^{\prime} \in A_{\leq} \text {, and } \alpha_{1}, \alpha_{2} \in A \text { satisfying } \\
& \left.\pi\left(\alpha^{\prime} \alpha_{1} \alpha_{2}\right)=h(w \perp)\right\} .
\end{aligned}
$$

Since $4 \mu<\varphi(w \perp) \leq(|w|+1) \cdot \mu$, we see that $|w|>3$. Hence, $R_{1,1}$ is a finite system of length-reducing rules. The given weight restrictions for $w \perp$ imply that the left-hand side of no rule of $R_{1,1}$ is a proper suffix of the left-hand side of any other rule of $R_{1,1}$. Further, the right-hand side $\alpha^{\prime} \alpha_{1} \alpha_{2}$ of a rule of $R_{1,1}$ is uniquely determined by the left-hand side, since the morphism $h$ is injective. Hence, there are no non-trivial overlaps between the left-hand sides of the rules of $R_{1,1}$.

$$
\begin{aligned}
R_{1,2}:= & \left\{w \alpha_{1}^{\prime} \rightarrow \alpha_{2}^{\prime} \alpha \mid w=a v \in \Sigma^{*} \text { for some } a \in \Sigma, \alpha_{1}^{\prime}, \alpha_{2}^{\prime} \in A_{\leq}\right. \\
& \text {and } \alpha \in A \text { such that }\left|h(v) \pi\left(\alpha_{1}^{\prime}\right)\right| \leq 2 \mu<\left|h(w) \pi\left(\alpha_{1}^{\prime}\right)\right| \leq 3 \mu \\
& \text { and } \left.\pi\left(\alpha_{2}^{\prime} \alpha\right)=h(w) \pi\left(\alpha_{1}^{\prime}\right)\right\} .
\end{aligned}
$$

Since $\left|\pi\left(\alpha_{1}^{\prime}\right)\right| \leq \mu, 2 \mu<\left|h(w) \pi\left(\alpha_{1}^{\prime}\right)\right|=\varphi(w)+\left|\pi\left(\alpha_{1}^{\prime}\right)\right|$ implies that $\varphi(w)>\mu$, which in turn yields $|w| \geq 2$. Hence, $R_{1,2}$ is a finite system containing only length-reducing rules. As above it follows that there are no non-trivial overlaps between the left-hand sides of the rules of $R_{1,2}$.

(1.3) Working from right to left the rules of the subsystems $R_{1,1}$ and $R_{1,2}$ replace suffixes $v \perp$ of $\bar{\perp} q_{0} w \perp$ by the compressed form $c \in A \leq \cdot A^{*}$ of $h(v \perp)$. The subsystem $R_{1,3}$ will be used to replace the remaining prefix $I q_{0} u$ such that the resulting string belongs to $\bar{A}^{*} \cdot A_{Q} \cdot A^{*}$, that is, it is the compressed 
form of a string $x \in(\Gamma \cup Q)^{*}$ satisfying $|h(x)| \equiv 0 \bmod 2 \mu$. Unfortunately, the initial configuration $\perp q_{0} w \perp$ may not satisfy this requirement. Therefore, if $\left|h\left(\perp q_{0} w \perp\right)\right| \equiv r \bmod 2 \mu$ for some $r \in\{1, \ldots, 2 \mu-1\}$, then instead of compressing this initial configuration, we compress the configuration $\perp u q v \perp$ that is obtained from $\perp q_{0} w \perp$ after $r$ steps of $M$. Then $|h(\perp u q v \perp)|=\left|h\left(\perp q_{0} w \perp\right)\right|-r \equiv 0 \bmod 2 \mu$, and hence, $h(\perp u q v \perp)$ can be encoded through a string $c \in \bar{A}^{*} \cdot A_{Q} \cdot A^{*}$ such that $\pi(c)=h(\perp u q v \perp)$.

In each step the sDTPDA $M$ can remove at most one symbol from the top of its second pushdown store. Thus, the first $2 \mu-1$ steps of the computation of $M$ on input $w$ depend only on the prefix $u$ of $w$ of length $2 \mu-1$. Hence, the rules of $R_{1,3}$ will encode all computations of $M$ of this form.

$$
\begin{aligned}
R_{1,3}:= & \left\{I{ }_{q_{0}} w \alpha^{\prime} \alpha_{1} \cdots \alpha_{n} \rightarrow \beta_{1} \cdots \beta_{m} \mid w \in \Sigma^{*}, \alpha^{\prime} \in A_{\leq}, \alpha_{1}, \ldots, \alpha_{n} \in A\right. \\
& \text { such that }\left|h(w) \pi\left(\alpha^{\prime}\right)\right| \leq 2 \mu, 2 \leq n \leq \mu, \text { where } n<\mu \\
& \text { implies that } \pi\left(\alpha^{\prime} \alpha_{1} \cdots \alpha_{n}\right) \in((\Gamma \backslash\{\perp\}) \cup\{\#\})^{*} \cdot h(\perp), \\
& \text { and } \beta_{1}, \ldots, \beta_{m} \in \bar{A} \cup A_{Q} \cup A \text { satisfy the following conditions: } \\
& \text { (i) } \beta_{1} \cdots \beta_{m} \in \bar{A}^{*} \cdot A_{Q} \cdot A^{*}, \\
& \text { (ii) } h(w) \pi\left(\alpha^{\prime} \alpha_{1} \cdots \alpha_{n}\right)=h(v) x \text { for some } v \in(\Gamma \backslash\{\perp\})^{*} \cdot\{\perp, \varepsilon\} \\
& \text { and } x \in(\Gamma \cup\{\#\})^{*} \text { satisfying }|x|<\mu, \text { and } \\
& \text { (iii) } \pi\left(\beta_{1} \cdots \beta_{m}\right)=h\left(u_{1} q_{1} v_{1}\right) x \text { for some } u_{1}, v_{1} \in \Gamma^{*} \text { and } q_{1} \in Q \\
& \text { such that } \perp q_{0} v r_{M}^{r} u_{1} q_{1} v_{1} \text {, where } r \in\{0,1, \ldots, 2 \mu-1\} \\
& \text { satisfies } \left.\left|h\left(\perp q_{0} w\right) \pi\left(\alpha^{\prime}\right)\right| \equiv r \bmod 2 \mu\right\} .
\end{aligned}
$$

If $\left(I q_{0} w \alpha^{\prime} \alpha_{1} \cdots \alpha_{n} \rightarrow \beta_{1} \cdots \beta_{m}\right) \in R_{1,3}$, then $m \in\{n, n+1, n+2\}$. Hence, $R_{1,3}$ is a finite system of length-reducing rules. It can easily be checked that there are no non-trivial overlaps between the left-hand sides of the rules of $R_{1,3}$.

The subsystem $R_{1}$ is now taken as $R_{1}:=R_{1,1} \cup R_{1,2} \cup R_{1,3}$. From the definitions given it follows immediately that there are no non-trivial overlaps between the left-hand sides of the rules of $R_{1}$.

(2) The subsystem $R_{2}$ simulates the computations of the sDTPDA $M$ on strings that represent compressed forms of configurations. Each application of a rule of $R_{2}$ simulates $2 \mu$ steps of $M$.

$$
\begin{aligned}
R_{2}:= & \left\{\alpha_{1} \cdots \alpha_{n} \gamma \alpha_{n+1} \cdots \alpha_{n+m} \rightarrow \beta_{1} \cdots \beta_{n+m} \mid \alpha_{1}, \ldots, \alpha_{n} \in \bar{A}, \gamma \in A_{Q},\right. \\
& \alpha_{n+1}, \ldots, \alpha_{n+m} \in A \text { such that } n, m \leq \mu+1, \text { where } \\
& 1 \leq n \leq \mu \text { implies that } \pi\left(\alpha_{1}\right) \text { has prefix } h(\perp), \\
& n=0 \quad \text { implies that } \pi(\gamma) \text { has prefix } h(\perp) \text { and } m \geq 2, \\
& 1 \leq m \leq \mu \text { implies that } \pi\left(\alpha_{n+m}\right) \text { has suffix } h(\perp), \text { and } \\
& m=0 \quad \text { implies that } \pi(\gamma) \text { has suffix } h(\perp) \text { and } n \geq 2, \\
& \beta_{1}, \ldots, \beta_{n+m} \in A \cup A_{Q} \cup A \text { such that } \beta_{1} \cdots \beta_{n+m} \in \bar{A}^{*} \cdot A_{Q} \cdot A^{*}, \\
& \pi\left(\alpha_{1} \cdots \alpha_{n} \gamma \alpha_{n+1} \cdots \alpha_{n+m}\right)=x_{1} h(u q v) x_{2} \text { for some } u, v \in \Gamma^{*}, q \in Q, \\
& x_{1} \in\{\#\}^{*}, x_{2} \in \Gamma \cdot\{\#\}^{*},\left|x_{1}\right|,\left|x_{2}\right|<\mu, x_{2} \notin h(\Gamma), \\
& \text { and } \pi\left(\beta_{1} \cdots \beta_{n+m}\right)=x_{1} h\left(u_{1} q_{1} v_{1}\right) x_{2} \text { for some } u_{1}, v_{1} \in \Gamma^{*}, q_{1} \in Q, \\
& \text { such that uqv and } u_{1} q_{1} v_{1} \text { are valid subconfigurations of } M \\
& \text { satisfying } \left.u q v{\vdash_{M}^{2}}_{M}^{2} u_{1} q_{1} v_{1}\right\} .
\end{aligned}
$$


The conditions on the integers $n$ and $m$ imply that $n+m \geq 2$. Further, all rules of $R_{2}$ are obviously length-reducing. Since $u q v$ and $u_{1} q_{1} v_{1}$ must be valid subconfigurations of $M, \perp$ can occur at most as the first and/or the last letter. Hence, the left-hand side of no rule of $R_{2}$ is contained in the left-hand side of another rule of $R_{2}$. Finally, the right-hand side of a rule of $R_{2}$ is uniquely determined by its left-hand side. Thus, there are no non-trivial overlaps between the left-hand side of the rules of $R_{2}$.

(3) The subsystem $R_{3}$ ends the simulation of computations of $M$.

$$
\begin{aligned}
& R_{3}:=\left\{\alpha_{1} \alpha_{2} \rightarrow Y \mid \alpha_{1}, \alpha_{2} \in \bar{A} \cup A_{Q} \cup A, \alpha_{1} \alpha_{2} \in \bar{A}^{*} \cdot A_{Q} \cdot A^{*}, \pi\left(\alpha_{1} \alpha_{2}\right)=\right. \\
&\left.h(\perp u q v \perp) \text { for some } u, v \in \Gamma^{*} \text { and } q \in Q, \text { and } \perp u q v \perp \vdash_{M}^{*} q_{f}\right\} \\
& \cup\left\{\alpha_{1} \alpha_{2} \rightarrow N \mid \alpha_{1}, \alpha_{2} \in \bar{A} \cup A_{Q} \cup A, \alpha_{1} \alpha_{2} \in \bar{A}^{*} \cdot A_{Q} \cdot A^{*}, \pi\left(\alpha_{1} \alpha_{2}\right)=\right. \\
&\left.h(\perp u q v \perp) \text { for some } u, v \in \Gamma^{*} \text { and } q \in Q, \text { and } \perp u q v \perp \vdash_{M}^{*} \perp q_{f}\right\} .
\end{aligned}
$$

Obviously, $R_{3}$ is a finite length-reducing system, and there are no non-trivial overlaps between the left-hand side of the rules of $R_{3}$.

Finally, we take $R:=R_{0} \cup R_{1} \cup R_{2} \cup R_{3}$. Then $R$ is indeed a finite stringrewriting system that contains length-reducing rules only. It is easily verified that there are no non-trivial overlaps between the left-hand sides of the rules of $R$. Hence, we see that $R$ is also confluent.

It remains to prove the following statements for all $w \in \Sigma^{*}$ :

(i) If $w \in L$, then $\perp q_{0} w \perp \rightarrow_{R}^{*} Y$.

(ii) If $w \notin L$, then $I q_{0} w \perp \rightarrow_{R}^{*} N$.

These statements show that the system $R$, together with the strings $t_{1}:=I q_{0}$ and $t_{2}:=\perp$ and the letters $Y$ and $N$, witnesses the fact that $L$ is a ChurchRosser decidable language. The proof of the statements above will be divided into several claims and their proofs. The first one follows immediately from the choice of the subsystem $R_{0}$.

Claim 1. For all $w \in \Sigma^{*}$ satisfying $\varphi(w) \leq 4 \mu-\varphi(\perp)$ the statements (i) and (ii) hold.

Hence, for the following considerations we can assume that the string $w \in \Sigma^{*}$ satisfies $\varphi(w)>4 \mu-\varphi(\perp)$, that is, $\varphi(w \perp)>4 \mu$.

Claim 2. Let $w \in \Sigma^{*}$ such that $\varphi(w \perp)>4 \mu$, and let $r \in\{0,1, \ldots, 2 \mu-1\}$ such that $\varphi\left(\perp q_{0} w \perp\right)=k \cdot 2 \mu+r$ for some $k \in \mathbb{N}$. Then there exist $\alpha_{1}, \ldots, \alpha_{k} \in$ $\bar{A} \cup A_{Q} \cup A$ satisfying the following conditions:

(i) $\alpha_{1} \alpha_{2} \cdots \alpha_{k} \in \bar{A}^{*} \cdot A_{Q} \cdot A^{*}$,

(ii) $\pi\left(\alpha_{1} \cdots \alpha_{k}\right)=h(\perp u q v \perp)$ for some configuration $\perp u q v \perp$ of $M$,

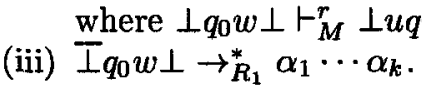

Proof. Let $w \in \Sigma^{*}$ satisfy $\varphi(w \perp)>4 \mu$, and let $k \in \mathbb{N}$ and $r \in\{0,1, \ldots, 2 \mu-1\}$ such that $\varphi\left(\perp q_{0} w \perp\right)=k \cdot 2 \mu+r>4 \mu$. The computation of $M$ starting from 
the initial configuration $\perp q_{0} w \perp$ either ends with the accepting configuration $q_{f}$ of weight $\varphi\left(q_{f}\right) \leq \mu$ or with the non-accepting configuration $\perp q_{f}$ of weight $\varphi\left(\perp q_{f}\right) \leq 2 \mu$. Hence, this computation consists of more than $2 \mu$ steps. Thus, there is a (uniquely determined) configuration $\perp u q v \perp$ of $M$ such that $\perp q_{0} w \perp \vdash_{M}^{r}$ $\perp u q v \perp$. Since $\varphi(\perp u q v \perp)=\varphi\left(\perp q_{0} w \perp\right)-r=k \cdot 2 \mu$, there exist $\alpha_{1}, \ldots, \alpha_{k} \in$ $\bar{A} \cup A_{Q} \cup A$ such that $\alpha_{1} \alpha_{2} \cdots \alpha_{k} \in \bar{A}^{*} \cdot A_{Q} \cdot A^{*}$ and $\pi\left(\alpha_{1} \cdots \alpha_{k}\right)=h(\perp u q v \perp)$. It follows easily from the definition of the rules of the system $R_{1}$ that $I q_{0} w \perp \rightarrow_{R_{1,1}}$ $w_{1} \rightarrow_{R_{1,2}}^{*} w_{2} \rightarrow R_{1,3} \alpha_{1} \alpha_{2} \cdots \alpha_{k}$ holds for some strings $w_{1}$ and $w_{2}$.

Claim 3. Let $\perp u q v \perp$ be a configuration of $M$ such that $\varphi(\perp u q v \perp)=s \cdot 2 \mu$ for some $s \geq 3$, and let $\alpha_{1}, \ldots, \alpha_{s} \in \bar{A} \cup A_{Q} \cup A$ such that $\alpha_{1} \cdots \alpha_{s} \in \bar{A}^{*} \cdot A_{Q} \cdot A^{*}$ and $\pi\left(\alpha_{1} \cdots \alpha_{s}\right)=h(\perp u q v \perp)$. If $\perp u q v \perp$ is reachable from an initial configuration, then there exist a configuration $\perp u_{1} q_{1} v_{1} \perp$ of $M$ and letters $\beta_{1}, \ldots, \beta_{s-1} \in \bar{A} \cup$ $A_{Q} \cup A$ such that the following conditions are satisfied:

(i) $\beta_{1} \beta_{2} \cdots \beta_{s-1} \in \bar{A}^{*} \cdot A_{Q} \cdot A^{*}$,

(ii) $\pi\left(\beta_{1} \beta_{2} \cdots \beta_{s-1}\right)=h\left(\perp u_{1} q_{1} v_{1} \perp\right)$,

(iii) $\perp u q v \perp \vdash_{M}^{2 \mu} \perp u_{1} q_{1} v_{1} \perp$, and

(iv) $\alpha_{1} \alpha_{2} \cdots \alpha_{s} \rightarrow_{R_{2}} \beta_{1} \beta_{2} \cdots \beta_{s-1}$.

Proof. Let $\perp u q v \perp$ be a configuration of $M$ such that $\varphi(\perp u q v \perp)=s \cdot 2 \mu$ for some $s \geq 3$. If $\perp u q v \perp$ is reachable from some initial configuration, that is, $\perp q_{0} w \perp \vdash_{M}^{*} \perp u q v \perp$ for some $w \in \Sigma^{*}$, then $\perp u q v \perp \vdash_{M}^{*} q_{f}$ or $\perp u q v \perp \vdash_{M}^{*} \perp q_{f}$, depending on whether $w \in L$ or $w \notin L$, respectively. Since the weight of the actual configuration decreases by 1 in each step, we see that there exists a unique configuration $\perp u_{1} q_{1} v_{1} \perp$ such that $\perp u q v \perp \vdash_{M}^{2 \mu} \perp u_{1} q_{1} v_{1} \perp$ and $\varphi\left(\perp u_{1} q_{1} v_{1} \perp\right)=\varphi(\perp u q v \perp)-2 \mu=(s-1) \cdot 2 \mu$. Hence, there exist (uniquely determined) $\beta_{1}, \beta_{2}, \ldots, \beta_{s-1} \in \bar{A} \cup A_{Q} \cup A$ satisfying $\beta_{1} \beta_{2} \cdots \beta_{s-1} \in \bar{A}^{*} \cdot A_{Q} \cdot A^{*}$ and $\pi\left(\beta_{1} \beta_{2} \cdots \beta_{s-1}\right)=h\left(\perp u_{1} q_{1} v_{1} \perp\right)$.

During the computation $\perp u q v \perp \vdash_{M}^{2 \mu} \perp u_{1} q_{1} v_{1} \perp$ a suffix $u^{\prime}$ of $u$ and a prefix $v^{\prime}$ of $v$ are involved that satisfy $\left|u^{\prime}\right|,\left|v^{\prime}\right| \leq 2 \mu$. Hence, this computation can be described completely by using a window of length $2 \mu+1+2 \mu=4 \mu+1$ that is placed on $\perp u q v \perp$ in such a way that the state symbol $q$ appears in the middle. The corresponding section of $h(\perp u q v \perp)$ is contained in a substring $\alpha_{1}^{\prime} \cdots \alpha_{n}^{\prime} \gamma^{\prime} \alpha_{n+1}^{\prime} \cdots \alpha_{n+m}^{\prime} \in \bar{A}^{*} \cdot A_{Q} \cdot A^{*}$ of $\alpha_{1} \alpha_{2} \cdots \alpha_{s}$ satisfying $n, m \leq \mu+1$. From the definition of the subsystem $R_{2}$ we see that each rule of $R_{2}$ just simulates $2 \mu$ steps of $M$ on a substring of this form. Hence, it follows that $\alpha_{1} \alpha_{2} \cdots \alpha_{s} \rightarrow_{R_{2}}$ $\beta_{1} \beta_{2} \cdots \beta_{s-1}$ holds.

Claim 4. Let $\perp u q v \perp$ be a configuration of $M$ such that $\varphi(\perp u q v \perp)=4 \mu$, and let $\alpha_{1}, \alpha_{2} \in \bar{A} \cup A_{Q} \cup A$ such that $\alpha_{1} \alpha_{2} \in \bar{A}^{*} \cdot A_{Q} \cdot A^{*}$ and $\pi\left(\alpha_{1} \alpha_{2}\right)=h(\perp u q v \perp)$. If $\perp u q v \perp$ is reachable from an initial configuration, then either $\alpha_{1} \alpha_{2} \rightarrow R_{3} Y$ or $\alpha_{1} \alpha_{2} \rightarrow_{R_{3}} N$. 
Proof. Let $\perp u q v \perp$ be a configuration of $M$ such that $\varphi(\perp u q v \perp)=4 \mu$, and let $\alpha_{1}, \alpha_{2} \in \bar{A} \cup A_{Q} \cup A$ such that $\alpha_{1} \alpha_{2} \in \bar{A}^{*} \cdot A_{Q} \cdot A^{*}$ and $\pi\left(\alpha_{1} \alpha_{2}\right)=h(\perp u q v \perp)$. If $\perp u q v \perp$ is reachable from some initial configuration, then $\perp q_{0} w \perp \vdash_{M}^{*} \perp u q v \perp$ for some $w \in \Sigma^{*}$. If $w \in L$, then $\perp u q v \perp \vdash_{M}^{*} q_{f}$, and if $w \notin L$, then $\perp u q v \perp \vdash_{M}^{*} \perp q_{f}$. Thus, either $\left(\alpha_{1} \alpha_{2} \rightarrow Y\right) \in R_{3}$ or $\left(\alpha_{1} \alpha_{2} \rightarrow N\right) \in R_{3}$.

We now verify that $R$ does indeed witness the fact that $L$ is a Church-Rosser decidable language. Let $w \in \Sigma^{*}$. If $\varphi(w) \leq 4 \mu-\varphi(\perp)$, then we see from Claim 1 that $\bar{\perp} q_{0} w \perp \rightarrow_{R} Y$ if $w \in L$, and $\bar{\perp} q_{0} w \perp \rightarrow_{R} N$, if $w \notin L$. Assume therefore that $\varphi(w)>4 \mu-\varphi(\perp)$. Then by Claim 2 there exist a configuration $\perp u_{1} q_{1} v_{1} \perp$ of $M$ and $\alpha_{1}, \alpha_{2}, \ldots, \alpha_{k} \in \bar{A} \cup A_{Q} \cup A$ such that

(i) $\alpha_{1} \alpha_{2} \cdots \alpha_{k} \in \bar{A}^{*} \cdot A_{Q} \cdot A^{*}$,

(ii) $\pi\left(\alpha_{1} \alpha_{2} \cdots \alpha_{k}\right)=h\left(\perp u_{1} q_{1} v_{1} \perp\right)$,

(iii) $\perp q_{0} w \perp \vdash_{M}^{*} \perp u_{1} q_{1} v_{1} \perp$, and

(iv) $\perp q_{0} w \perp \rightarrow_{R}^{*} \alpha_{1} \cdots \alpha_{k}$.

If $k>2$, then Claim 3 applies. Hence, there are configurations $\perp u_{i} q_{i} v_{i} \perp$ of $M$ and strings $\delta_{i} \in \bar{A}^{*} \cdot A_{Q} \cdot A^{*}, i=2, \ldots, k-1$, such that $\perp u_{i-1} q_{i-1} v_{i-1} \perp \vdash_{M}^{2 \mu}$ $\perp u_{i} q_{i} v_{i} \perp, \pi\left(\delta_{i}\right)=h\left(\perp u_{i} q_{i} v_{i} \perp\right), \alpha_{1} \cdots \alpha_{k} \rightarrow_{R} \delta_{2} \rightarrow_{R} \ldots \rightarrow_{R} \delta_{k-1}$, and $\left|\delta_{i}\right|=$ $k-i+1$ for all $i=2, \ldots, k-1$. Finally, $\left|\delta_{k-1}\right|=2$ implies that $\delta_{k-1} \rightarrow_{R} Y$ or $\delta_{k-1} \rightarrow_{R} N$ by Claim 4. From the definition of $R_{3}$ we see that the former is the case if and only if $w \in L$. Thus, for $w \in L$, we have $I q_{0} w \perp \rightarrow_{R}^{*} \alpha_{1} \cdots \alpha_{k} \rightarrow_{R}$ $\ldots \rightarrow_{R} \delta_{k-1} \rightarrow_{R} Y$, and for $w \notin L$, we have $I q_{0} w \perp \rightarrow_{R}^{*} \alpha_{1} \cdots \alpha_{k} \rightarrow_{R} \ldots \rightarrow_{R}$ $\delta_{k-1} \rightarrow_{R} N$. This completes the proof of Theorem 5 .

From Theorem 5 we obtain our main result.

Corollary 6. The three language classes $\mathrm{CRDL}, \mathrm{CRL}$, and GCRL coincide.

Thus, the Church-Rosser languages are indeed the deterministic variants of the growing context-sensitive languages.

\section{Closure Properties}

In this section we summarize the known closure and non-closure properties of the class CRL and we prove two new non-closure properties, which, however, were already announced by Buntrock and Otto [7].

From the definition of the class CRDL we immediately obtain the following result.

Proposition 7. The class of Church-Rosser languages is closed under complementation, that is, if $L \subseteq \Sigma^{*}$ is a Church-Rosser language, then so is the language $\bar{L}:=\Sigma^{*} \backslash L$.

From the characterization of the class GCRL through the shrinking DTPDA we can conclude the following closure properties. 


\section{Proposition 8.}

(a) The class $\mathrm{CRL}$ is closed under intersection with regular languages, that is, if $L \in \mathrm{CRL}$ and $L_{1}$ is a regular language, then $L \cap L_{1} \in \mathrm{CRL}$.

(b) The class CRL is closed under inverse morphisms, that is, if $L \subseteq \Sigma^{*}$ is in $\mathrm{CRL}$ and $h: \Delta^{*} \rightarrow \Sigma^{*}$ is a morphism, then $h^{-1}(L) \in \mathrm{CRL}$.

Finally, from [11] we recall the following closure properties.

\section{Proposition 9.}

(a) $\mathrm{CRL}$ is closed under reversal, that is, if $L$ is a Church-Rosser language, then so is the language $L^{\sim}:=\left\{w^{\sim} \mid w \in L\right\}$.

(b) $\mathrm{CRL}$ is closed under left quotient and right quotient with a single string, that is, if $L \subseteq \Sigma^{*}$ is a Church-Rosser language and $z \in \Sigma^{*}$, then $L /\{z\}=\{w \in$ $\left.\Sigma^{*} \mid w z \in L\right\}$ and $\{z\} \backslash L:=\left\{w \in \Sigma^{*} \mid z w \in L\right\}$ are Church-Rosser languages, too.

In [12] it is shown that the class CRL is a basis for the recursively enumerable languages. Further, it is shown by Buntrock in [4] that the closure of the class GCRL (= CRL) under $\varepsilon$-free morphisms yields the class GCSL. Hence, we obtain the following non-closure properties.

Proposition 10. The class CRL is neither closed under projections nor under E-free morphisms.

The Gladkij language $L_{\mathrm{Gl}}:=\left\{w \phi w^{\sim} \phi w \mid w \in\{a, b\}^{*}\right\}$ is a context-sensitive language that is not growing context-sensitive $[9,1,7]$. Now $L_{\mathrm{Gl}}$ can be written as $L_{\mathrm{Gl}}=L_{1} \cap L_{2}$, where $L_{1}:=\left\{w \phi w^{\sim} \phi z \mid w, z \in\{a, b\}^{*}\right\}$ and $L_{2}:=\left\{w \phi z \phi z^{\sim} \mid\right.$ $\left.w, z \in\{a, b\}^{*}\right\}$. Obviously, $L_{1}$ and $L_{2}$ are both deterministic context-free, and hence, they are both Church-Rosser languages. Since $L_{1} \cap L_{2} \notin \mathrm{GCSL}$, we have $L_{1} \cap L_{2} \notin C R L$. This shows the following.

Proposition 11. The class CRL is neither closed under intersection nor under union.

\section{Conclusion}

We have shown that the three language classes CRDL and CRL of [11] and GCRL of [7] coincide. Because of the characterization of the latter class through the deterministic variant of the shrinking TPDA [7] this class of languages can be considered as the class of 'deterministic growing context-sensitive languages'. Based on these characterizations we have obtained some closure properties and some non-closure properties for the class of Church-Rosser languages. However, many questions regarding closure and non-closure properties remain open. Also it 
remains the question of whether or not the language $L_{0}:=\left\{w w^{\sim} \mid w \in\{a, b\}^{*}\right\}$ is a Church-Rosser language.

Finally, based on the fact that the classes CFL and CRL are incomparable under set inclusion, we obtain the following undecidability result from McNaughton et al [11].

\section{Proposition 12.}

(a) The emptiness and the finiteness problems for Church-Rosser languages are undecidable in general.

(b) It is undecidable in general whether a given context-free language is a ChurchRosser language.

(c) It is undecidable in general whether a given Church-Rosser language is context-free.

\section{References}

1. R.V. Book. Grammars with Time Functions. PhD thesis, Harvard University, Cambridge, Massachusetts, February 1969.

2. R.V. Book. Confluent and other types of Thue systems. J. Association Computing Machinery, 29:171-182, 1982.

3. R.V. Book and F. Otto. String-Rewriting Systems. Springer-Verlag, New York, 1993.

4. G. Buntrock. Wachsende kontext-sensitive Sprachen. Habilitationsschrift, Fakultät für Mathematik und Informatik, Universität Würzburg, July 1996.

5. G. Buntrock and K. Loryś. On growing context-sensitive languages. In W. Kuich, editor, Proc. of 19th ICALP, Lecture Notes in Computer Science 623, pages 77-88. Springer-Verlag, Berlin, 1992.

6. G. Buntrock and K. Loryś. The variable membership problem: Succinctness versus complexity. In P. Enjalbert, E.W. Mayr, and K.W. Wagner, editors, Proc. of 11th $S T A C S$, Lecture Notes in Computer Science 775, pages 595-606. Springer-Verlag, Berlin, 1994.

7. G. Buntrock and F. Otto. Growing context-sensitive languages and Church-Rosser languages. In E.W. Mayr and C. Puech, editors, Proc. of 12th STACS, Lecture Notes in Computer Science 900, pages 313-324. Springer-Verlag, Berlin, 1995.

8. E. Dahlhaus and M. Warmuth. Membership for growing context-sensitive grammars is polynomial. J. Computer System Sciences, 33:456-472, 1986.

9. A.W. Gladkij. On the complexity of derivations for context-sensitive grammars. Algebri i Logika Sem., 3:29-44, 1964. In Russian.

10. J.E. Hopcroft and J.D. Ullman. Introduction to Automata Theory, Languages, and Computation. Addison-Wesley, Reading, M.A., 1979.

11. R. McNaughton, P. Narendran, and F. Otto. Church-Rosser Thue systems and formal languages. J. Association Computing Machinery, 35:324-344, 1988.

12. F. Otto, M. Katsura, and Y. Kobayashi. Cross-sections for finitely presented monoids with decidable word problems. In H. Comon, editor, Rewriting Techniques and Applications, Lecture Notes in Computer Science 1232, pages 53-67. SpringerVerlag, Berlin, 1997. 